\title{
Rotation curve of our Galaxy and field galaxies
}

\author{
D. Russeil, O. Garrido, P. Amram and M. Marcelin \\ LAM, Observatoire de Marseille, 2 place Leverrier, 13004 Marseille, \\ France
}

\begin{abstract}
We present a database of galaxies rotation curve (from $\mathrm{H} \alpha$ observations), including our Galaxy.
\end{abstract}

\section{Introduction}

N-body simulations of cosmological evolution have now reached a sufficient resolution to predict dark halos density profiles down to the innermost parts of spiral galaxies. Moreover, the mass distribution of the luminous and dark matter deduced from multi-component mass models is strongly constrained by the inner slope of the rotation curves only correctly drawn by 2D velocity fields (Amram \& Garrido 2001). Correct inner shape rotation curves allow disentangling cosmological scenarios (Blais-Ouellette et al. 2001). Such data are provided by $\mathrm{H} \alpha$ velocity fields. Such high-resolution fields are complementary to HI velocity fields mapping the outer galactic regions but suffer from a lack of emission in the inner regions.

Up to now, there has not been a homogeneous sample of nearby and isolated spirals with a large range of morphological types and luminosities allowing statistical and individual studies.

The Marseille Observatory galactic plane and the GHASP surveys will offer such data for the Milky Way as well as for nearby field galaxies. This database will constitute a unique and homogeneous sample of velocity fields to be used as a reference sample.

\section{The GHASP (Gassendi HAlpha Survey of sPirals) Survey}

The survey is providing the $\mathrm{H} \alpha$ velocity fields of about 200 spiral and irregular galaxies (Garrido et al. 2002, 2003). The observations, made at the Observatoire de Haute-Provence $1.93 \mathrm{~m}$ telescope, use a scanning Fabry-Perot interferometer and a photon counting camera (field of view $5.8 \mathrm{arcmin}^{2}$, pixel size $0.68 \mathrm{arcsec}$ ). The sample is made up of local and isolated galaxies covering a large range of luminosity and morphological types. Such a sample will allow us to constrain internal region kinematics and dynamics as well as mass distribution (Carignan 1985; Amram \& Garrido 2001) and to compare the velocity fields of galaxies in various environments, at different stage of evolution and at higher redshifts. In addition, the data are going to complement the radio survey WHISP (Westerbrok survey of HI SPiral galaxies). 


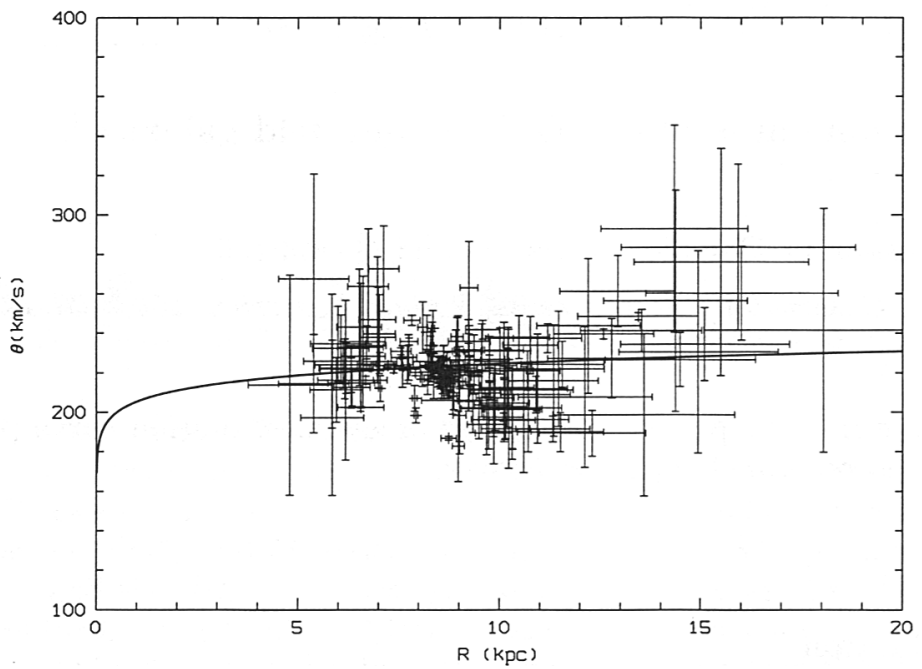

Figure 1. Circular rotation vs. distance of star-forming complexes. The superimposed solid line is the Brand and Blitz (1993) mean rotation curve.

\section{The Marseille $\mathrm{H} \alpha$ survey of the Southern Galactic plane}

The survey has been led using a scanning Fabry-Perot and a photon counting detector attached to the $36 \mathrm{~cm}$ telescope (field of view $39 \operatorname{arcmin}^{2}$, pixel size 9 arcsec) installed at La Silla Observatory. Such an instrument provides velocity information throughout the observed field. The survey is limited to the starforming complexes located in the $4^{\text {th }}$ quadrant of the Galactic plane. The main results consist of the optical detection and internal motion study of a large number of HII regions and the following of the warm interstellar medium diffuse emission. This diffuse emssion is always detected, and has similar velocity to the discrete HII regions. This survey acted in an essential role in the delimiting, listing and distance determination of the star-forming complexes in our Galaxy (e.g., Georgelin et al. 2000) and hence the rotation curve tracing (Russeil 2003). The mean rotation curve we obtain appears flat and departures from circular motion can be noted despite the large error bars (Fig. 1).

\section{References}

Amram, P., \& Garrido, O. 2001, ASP Conf. Ser. Vol. 282, 103

Blais-Ouellette, S., Amram, P., \& Carignan, C. 2001, AJ, 121, 4

Brand, J., \& Blitz, L. 1993, A\&A, 275, 67

Carignan, C. 1985, ApJ, 299, 59

Garrido, O., Marcelin, M., Amram, P., et al. 2002, A\&A, 387, 821

Garrido, O., Marcelin, M., Amram, P., et al. 2003, A\&A, 399, 51

Georgelin, Y. M., Russeil, D., Amram, P., et al. 2000, A\&A, 357, 308

Russeil, D. 2003, A\&A, 397, 133 